\title{
Occlusion Detection and Handling: A Review
}

\author{
Himanshu Chandel \\ Bahra University \\ Waknghat, Shimla
}

\author{
Sonia Vatta \\ Bahra University \\ Waknaghat, Shimla
}

\begin{abstract}
Object tracking and detection is a classical research area in the field of computer vision from decades. Numerous kinds of applications are dependent on the area of object detection, such as advance driving assistance system, traffic surveillance, scene understanding, autonomous navigation etc. Many challenges still exist while detecting an object such as illusion, low visibility, cast shadows and most importantly occlusions of object. Occlusions occur under two categories, firstly its, self-occlusion which means that, from a certain viewpoint, one part of an object is occluded by another part. Secondly, its inter-object occlusion which means when two objects being tracked occlude each other. We will review various occlusion handling methods that involved single and multiple cameras according to their application. In short, the objective of this paper is to deliberate in detail the problem of occlusion in object tracking and provide a concise review for the problem of occlusion handling under different categories and identify new trends.
\end{abstract}

\section{General Terms}

Computer vision, Driverless Cars, Algorithms, HOG, DPM.

\section{Keywords}

Occlusion; tracking; scene; vision; unmanned; car.

\section{INTRODUCTION}

In computer vision object detection is an active area of research since decades. The interest is due to various applications such as advance driving assistance system [1], surveillance [2], scene understanding [3]. The core challenge and the basic step in tracking is to accurately detect the object in different environments, but due to complex backgrounds, weather conditions, cast shadows and occlusions it becomes difficult to track an object. In this paper we will be targeting the problem of occlusion handling as it can't be avoided in applications of object tracking. Many computer vision algorithms suffer due to the presence of occluded objects in a scene. The region, which is occluded though, depends on the camera viewpoint. In some scenarios angle of the camera can define which part is occluded and which one is not, hence minimization approach, temporal selection, graph cut method and sum of squared distance are followed for handling the same problem of occlusion [15]. The very basic technique for predicting occlusion in tracking was started from the geometric information [28, 29]. One solution to occlusion problem is to use the fusion of multiple cameras to determine depth and further estimating the occluded part [30]. Depth estimation itself is a challenging problem since local features alone are insufficient to estimate depth at a point and needs to consider the global context of an image, hence a polynocular stereo algorithm is used for occlusion handling [33]. Rosales and Sclaroff presented the method of temporary and trajectory prediction for the same problem [31].Geometry of a scene plays a vital character in handling occlusion, the technique of epipolar lines and disparity map are used by Davi et.al to get the robust solution [35]. Little and Gillet put the technique of suppression using ordering constraint [36]. Identifying the occlusion regions, by defining the two occlusion maps, that is occlusion map1 showing parts of image 1 , that are not visible in image 2 and similarly occlusion map 2 in image 2 , while the displacement field between the images act as a key part in this proficiency [37].

Occlusion is the basic element that limits the information in an image. In vision based systems researchers commonly deal with binocular or stereo vision. Binocular is the process of obtaining depth information from a pair of cameras. In stereo vision occlusion occurs when a portion of the picture visible on one image is occluded in the other by the scene itself or, a section of the scene near the image boundary moves out of the field of persuasion on the other picture. The core challenge in tracking is to accurately detect the object in a wide range of environments such as sunny and rainy conditions in driving assistance systems. For autonomous navigation systems, whether its unmanned ground vehicles (UGV), unmanned aerial vehicles (UAV) or unmanned underwater vehicles (UUV), vision is playing a crucial role in path planning and autonomous navigation. In comparison to range sensors such as LIDAR and SONAR, camera is gaining attention for vision based systems as cameras not only deliver geometry but also provide rich appearance information. One of the simplest method for detecting occlusion is cross checking and extrapolation [24]. Mehmood, Nawaz and Rao in addition to the Bhattacharya Coefficient threshold used the normalized cross correlation method for handling occlusion. The same technique was followed further, Normalized cross correlation is used at every step followed by subtracting the mean and dividing the standard deviation [17, 18]. Asymmetric information as well as geometric, photometric constraints and robust cost aggregation for occluded candidates have also been used for handling occlusions [19]. The main objective of [20] is to detect and handle occlusion inside a tunnel and on real road using video as an input. In this paper Position of trajectories is estimated using Relative descriptive histogram, which is an extended approach of HOG [10]. Image segmentation is the core step for various computer vision algorithms and the same technique has been adopted by Enzweiler, Eigenstetter, Schiele and Gavrila for handling occlusion using Mean shift algorithm. Artificial neural networks are trained on intensity depth and motion features. The expert weights are computed that are related to the degree of visibility of the associated component [21]. In stereo vision occlusion is the classical research problem as images are captured from different angles and some pixels are available only in one image. The same approach is used for handling occlusion that is left and right disparity maps are obtained with the help of optimization based on modified constant space belief propagation [22, 23]. Scene understanding started with the dream of constructing machines that can see like humans to understand general principles and current situations from images, but it has become lot more extensive than that. 
Applications such as image search engines, autonomous driving, computational photography, vision for graphics, human machine interaction, were unexpected and other applications keep rising as scene understanding technology develops. As a center setback of high level computer vision, while it has enjoyed some great success in the past 50 years, a great deal more is needed to achieve a perfect understanding of visual scenes. Christian Wojek concentrates on reliable multi-object tracking from a moving platform in challenging real-world scenes with object - object occlusion using SVM (Support vector machine) and DPM (Deformable Parts Model) which is good in long term occlusion, basically on human detection [25]. Next, the goal of Fehrman and McGoughis to fully remove the occluding objects from the image and look at what is behind them using the canny edge detector function and disparity map generation [27]. Kalman filtering is the classical approach used widely for tracking. Appearance models are used for handling occlusions in each track showing how the object appears in an image [32]. Niknejad, Kawano, Oishi, Mita uses two layers classifiers using Conditional Random Field (CRF) and Deformable Parts Model (DPM) without any knowledge from previous frames [26]. Novel approach has been adopted by $\mathrm{Zhang}, \mathrm{Xu}$, Beaugendre and Goto for the same problem of handling occlusions in human tracking based on Kannade-Lucas Tomasi (KLT) method, they have used PETS and CAVIAR dataset.

We further classify the various methods and techniques used for occlusion handling on the basis of their applications in scene understanding. The applications are divided into four sections in section 2, we explain about the techniques used for car occlusion in realistic multiple traffic and street scenes followed by human occlusion in various scenes in section 3 . Then, in section 4 , diverse real world occluded scenes are reported and in section 5, miscellaneous applications are discussed. Finally, conclusion will be presented in section 6 .

\section{CAR OCCLUSION IN STREET SCENES}

Due to increase of traffic on roads, intelligent traffic surveillance systems are being implemented in various countries for highway monitoring and city road management system. Traditionally, shadow detection techniques have been employed for removing shadows from the background and foreground, but Sadeghi \& Fathy used it as a novel feature for vehicles detection and occlusion handling. They have used photometric characteristic of darkest pixels of strong shadows in traffic images for occlusion handling [3]. Multilevel framework is adopted by Zhang, Jonathan $\mathrm{Wu}$, Yang and Wang for handling car occlusion in traffic; firstly occlusion is detected by evaluating the compactness ratio and interior distance ratio of vehicles, and then the detected occlusion is handled by removing a cutting region of occluded vehicles. On interframe level, occlusion is detected by performing subtractive clustering on the motion vectors of vehicle. Next comes the tracking level, occluded vehicles are tracked by using a bidirectional occlusion reasoning mechanism [4]. The same problem of occlusion was handled with the help of geometric occlusion patterns and occlusion mask. The results are quite interesting as for heavily occluded vehicles that are only $30-60 \%$ visible, $83.1 \%$ of the occlusions are correctly predicted on inner city street scenes datasets with different levels of occlusion [11]. In latest approaches for handling car occlusion, different classifiers are used with the help of foreground and background segmentation. Straw, Hasler and Wersing uses car detection technique based on an analytical feature framework to improve the detection of occluded objects. In this work, the new classifier is trained on the background segments by using cars with an occlusion up to $80 \%$ [8]. In another work for handling structured occlusion in traffic scenes, SIFT descriptors are computed for an input image and holistic classifiers are used. These classifiers are subdivided into three vertical parts (C_3 split) and each classifier is trained with car views. Occlusion is handled in two stages with a rate of $0 \%$ and $<80 \%$ and as an extension, depth information for individual car parts helps the classifier to reason about typical occlusion patterns [9]. Fei $\mathrm{Wu}$ et al. uses an access for handling occlusion inside a tunnel road and on real roads. According to them, partial occlusion still occurs, but the trajectory can handle this situation and tracks the vehicles well. Firstly, older trajectory is estimated and the estimated position of tracked vehicle is marked with gray. After updating older trajectories, newly generated trajectory estimates the new position using relative discriminative histogram of oriented gradients particle filter (RDHOGPF). Magnitudes are set to zero in the gray region using

$M(u, v)=\left\{\begin{array}{c}0 \\ M(u, v)\end{array}\right.$ if $F(u, v) \sim=1$

where $\mathrm{u}$ and $\mathrm{v}$ are the horizontal and vertical coordinates, respectively, $\mathrm{M}(\mathrm{u}, \mathrm{v})$ and $\mathrm{F}(\mathrm{u}, \mathrm{v})$ are the respective magnitude and foreground values in the normalized $32 \times 32$ image [20]. Pang, Lam and Yang propose a novel method for handling occlusion, which is tested on monocular traffic image sequences. They have adopted the texture based segmentation and deformable modeling method which solves most of the occlusion cases involving two vehicles but fails in severe occlusion [38]. Qing, Hoang and Jo deals with the problem of, same color vehicle occlusion as shown in figure1, using Bhattacharya distance and local edge image projection, for the separation of each occluded vehicle. For measuring the color similarity authors have used the bhattacharya distance between two color histograms of occluded vehicles [39].

\section{PEDESTRIAN OCCLUSION IN DIVERSED SCENES}

Occlusion is a challenging problem for tracking people under various conditions. Human detection is very important in video surveillance and intelligent vehicles such as UGV's and UAV's. Due to inconsistent manifestation and extensive series of poses which humans can espouse, detection of humans is a challenging task whether in a picture or a video. In a real environment, noteworthy amount of partial occlusion occurs as pedestrians move in the immediacy of other objects. Published literature on tracking system development is mostly focused on tracking an object moving in indoor environments. The remarkable progress in human detection and long-term tracking has allowed to detect and track numerous people concurrently in intricate scenes. Yet, systems are still relentlessly challenged by partial and full occlusions, which occur commonly in scenes of realistic complexity.

Rosales and Sclaroff reported a technique based on current 3D estimates of velocity and position to predict future locations of objects. If a collision is detected in objects, assumption is made that occlusion is likely to happen if the current estimation of error is given by Extended Kalman Filter (EKF). The occlusion detection routine predicts future locations of objects, based on current estimates of $3 \mathrm{D}$ velocity and position. By using estimated bounding box parameters and the binary maps for the objects being tracked, occlusion is predicted, detected and handled by estimating positions and 
velocities followed by applying these estimates to the image plane [31].

Chang, Gong and Ong attempted to handle the occlusion with multiple cameras. In this paper, tracking condition is divided in two parts: one camera occlusion (OCO) and two camera occlusion (TCO). In OCO, geometric and recognition based methods are used with the help of various cues such as epipolar line, landmarks, apparent color and height [30]. Condensation algorithm [30, 6] was used to track predicted joint features of the subject. $\mathrm{Hu}$ and Tan use the approach of Bayesian network for solving occlusion in tracking of people. The occlusion relation is categorized by $\phi \mathrm{t} \in\{0,1,2\}$, where $\phi \mathrm{t}=0$ indicates no occlusion, $\phi \mathrm{t}=1$ indicates $\mathrm{A}$ occludes $\mathrm{B}$, and $\phi \mathrm{t}=2$ indicates $\mathrm{B}$ occludes A [30]. Wang, Han and Yan developed a novel and impactful method for human detection and handling partial occlusion using the combination of Histogram of Gradient (HOG) and Local Binary Pattern (LBP). For scanning, whole windows global detector is used and for local regions, part detectors are used. With the help of the augmented HOG-LBP feature and the global-part occlusion handling method, they have achieved a detection rate of $91.3 \%$ with FPPW $=10-6,94.7 \%$ with $\mathrm{FPPW}=10-5$, and $97.9 \%$ with $\mathrm{FPPW}=10-4$ on the INRIA and Pascal dataset [10].

Vision based autonomous navigation is becoming more and more popular in unmanned vehicles, whether its air, ground or water. There are various issues and difficulties in vision based navigation systems such as weather conditions, cast shadows, strong headlights and complex backgrounds. One of the main setback in navigating the vehicle autonomously is occlusion caused by other vehicles and various objects. Ess, Schindler, Leibe and Gool have reported an approach for autonomous navigation and path planning, but most importantly, they have targeted on multiperson detection and targeting. Firstly, they generate the occlusion map that contains the regions occluded by both pedestrians and static obstacles. Secondly, Extended Kalman Filter (EKF) increases the chances of finding the object once it becomes visible again. In figure 2, the boxes are color coded to show tracked identities, darker boxes indicate objects in occlusion [12].

Enzweiler, Eigenstetter, Schiele and Gavrila uses the expert weights that are related to the degree of visibility for handling partial occlusion. The main objective of the reported study is pedestrian classification using images as input. The component based expert classifiers are trained on intensity depth and motion features which form the core of the used framework. Mean-shift segmentation with depth and motion is applied online for recovering occlusion dependent weights [21].

Stereo vision is a good approach for handling occlusions but Wojek, Walk, Roth and Schiele, have reported a monocular vision based scene understanding on the ETH-PedCross2 dataset. Multi-object tracking from a moving platform in challenging real-world scenes and object-object occlusion for human detection in ETH-PedCross2 have been achieved using seven detector components, deformable parts model and support vector machine (SVM). The reported technique was proved to be good for handling long term occlusions [25]. Hu and Tan uses the Bayesian Network approach for handling occlusions. Human model is compared with 2D ellipse model and tracking process is done using the condensation algorithm [6].

\section{DIVERSE REAL WORLD OCCLUDED SCENE}

In stereo images, to deal with occlusion, Kang, Szeliski and Chai uses two approaches. Firstly, they used adaptive windows and selected a temporal subset of the frames to match at each pixel. For explicitly modeling occluded regions, Global (MRF) minimization based on graph cuts was used as the second approach [15]. Sun, Li, Kang and Shum handled the same problem of occlusion using an asymmetric stereo matching model with visibility constraint. Two steps are considered; first the disparity map was deduced taking into consideration the occlusion map of another view. Then concluding the occlusion map in one view from the disparity map of another view. Linear support vector machine and segmentation by the mean-shift approach is also used in handling occlusions [13]. Many of the algorithms are designed to deal with short-duration partial occlusion, but they fail at severe occlusions and when a partial occlusion lasts for a long time. Yang, $\mathrm{Li}$, Pan and $\mathrm{Li}$ used an approach for multiple object tracking in dynamic scenes. The developed technique which has the ability to cope with long-duration and complete occlusion without a prior knowledge about the shape or motion of objects. Experiments are implemented on CAVIAR and PETS dataset in complex indoor and outdoor environments. The states of objects are separated into three parts: before, during and after occlusion. This Real time system uses video as an input and performs object segmentation using merging and splitting technique [18].

Object tracking has various applications in computer vision. $\mathrm{Pan}$ and $\mathrm{Hu}$ propose content adaptive progressive occlusion analysis algorithm for object tracking with occlusion handling. The entire Region of interest is determined, then the occlusion status of the block containing undetermined pixels are analyzed and finally all such blocks are scanned [5]. Ramik, Sabourin, Moreno and Madani developed an intelligent vision system that has the ability to learn autonomously individual objects present in a real environment without using human labeled data. Visual saliency is the nucleus of this paper for detection and recognition. SURF is used for object recognition as well as occlusion [14]. For handling occlusion while tracking, Guan, Chen, Yang and $\mathrm{Wu}$ implemented the occlusion handling component in three stages: occlusion detection, person re identification and tracklet generation. Before and after occlusion information is used to model the association cost matrix to re-identify persons under strong occlusion [17]. Chen, Li, D. Zhao and Q. Zhao gives a remarkable model shown in figure 3 for handling occlusion, inspired by the human perception of occlusion, further adding adaboost algorithm. They have used five dimensional region based cues; semantic, position, compactness, shared boundary and junction cue on rural artificial and outdoor scenes [40].

\section{MISCELLEANOUS}

The Weng, Ahuja and Huang reported an approach for detecting occlusions in 1988 by defining two occlusion maps, whose working is shown in figure4. Black areas represent occlusion regions. The displacement field from image 2 to image 1, without occlusion information is determined with the help of computed occlusion map1. Some of the areas in image 1 that have not been matched are occluded in image 2 and are mapped in occlusion map 1 [37]. When the location of the occluded region depends on the viewpoint, then the match suppression technique is used for occlusion handling [36]. Occlusions are the regions in one image that have no match in the other image. Geiger, Ladendorf and Yuille use the 
technique of stereo with an occlusion process $\mathrm{O}^{\mathrm{L}}$ and for the right $\mathrm{O}^{\mathrm{R}}$ such that: [35]

$$
\begin{aligned}
& \mathrm{O}_{\mathrm{l}}^{\mathrm{L}}(\mathrm{M})=1-\sum_{\mathrm{r}=0}^{\mathrm{N}-1} \mathrm{M}_{\mathrm{L}, \mathrm{R}} \\
& \mathrm{O}_{\mathrm{r}}^{\mathrm{R}}(\mathrm{M})=1-\sum_{\mathrm{l}=0}^{\mathrm{N}-1} \mathrm{M}_{\mathrm{L}, \mathrm{R}}
\end{aligned}
$$

The further disparity field is defined as for the left eye $\mathrm{D}^{\mathrm{L}}$ and the other for right eye $\mathrm{D}^{\mathrm{R}}$ by:

$$
\begin{array}{ll}
\mathrm{D}_{\mathrm{l}}^{\mathrm{L}}(\mathrm{M})=1-\sum_{\mathrm{r}=0}^{\mathrm{N}-1} \mathrm{M}_{\mathrm{L}, \mathrm{R}}(\mathrm{r}-\mathrm{l}) & \text { if } \mathrm{O}_{\mathrm{l}}^{\mathrm{L}}=0 \text { (4) } \\
\mathrm{D}_{\mathrm{r}}^{\mathrm{R}}(\mathrm{M})=1-\sum_{\mathrm{l}=0}^{\mathrm{N}-1} \mathrm{M}_{\mathrm{L}, \mathrm{R}}(\mathrm{r}-\mathrm{l}) & \text { if } \mathrm{O}_{\mathrm{r}}^{\mathrm{R}}=0 \text { (5) }
\end{array}
$$

Wunsch and Hirzinger used Kalman Filtering for estimating object velocity and acceleration. Geometric information was used for predicting occlusions [28]. Lathuiliere and Hervze handled the occlusion in application of hand posture. An occlusion validation algorithm was proposed which consisted of three parts: self-occlusion, sphere occlusion and cylinder occlusion [29]. Egnal and Wildes used five different algorithms for the purpose of detecting occlusion. Bimodality (BMD) and Match Goodness Jumps (MGJ) are the first two algorithms that detect occlusion boundaries and the rest are Left-Right Checking (LRC), Ordering (ORD) and occlusion constraint (OCC) [24]. Min and Sohn used geometric and photometric constraints for handling occlusion. Disparity map was estimated before asymmetric occlusion detection and the set occluded pixels were detected by the same technique [19]. From the dataset of Tsukuba, Venus, Teddy and Cone, images have been used by Jang and Hoe for handling occlusion using warping constraint and cross check constraint techniques. In this algorithm, the authors indicated that the pixels with largest disparity values are selected as visible pixels and rest matching pixels become occluded [22,23].

\section{CONCLUSION}

This paper provides a summarizing study for handling occlusions under different scenarios. We have tried to show the various Image processing, Computer vision and Machine Learning techniques for the same problem of occlusion. When both the objects occluded and occluder are being tracked by the same algorithm, it does not perform well in handling occlusions. DPM alone fails to handle occlusion, many methods have been used on various datasets, but still optimal solution is not there to handle occlusions in every scene. Lot of research has to be done to make it more accurate. The best feature we can conclude is shape as it helps to match occluded part of the object with the same object in the next frame. Various methods under stereo vision are showing some good progress in handling occlusions. Occlusion is a challenging problem in object detection and when it comes to stereo matching, it can't be neglected at all. Estimating disparity and occlusion by using disparity and occlusion maps is the classical solution of this problem and till date we don't have $100 \%$ accuracy in handling occlusions under various scenarios. Geometric patterns with the combination of machine learning is playing an effective role for handling occlusions. HOG has gained attention for object detection but when it comes to handling occlusion results are still unsatisfactory. Models inspired by human perception can lead to satisfactory occlusion handling results. In the end, we would conclude it in two points, there is still a large gap between human perception and occlusion reasoning. Lastly, if the detected features are better, the occlusion estimation will be better.

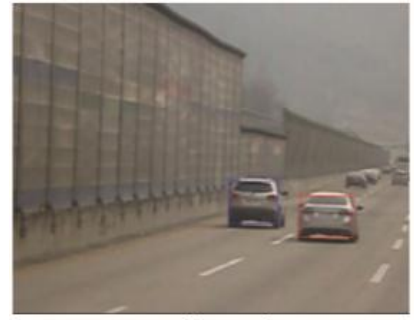

Frame 1

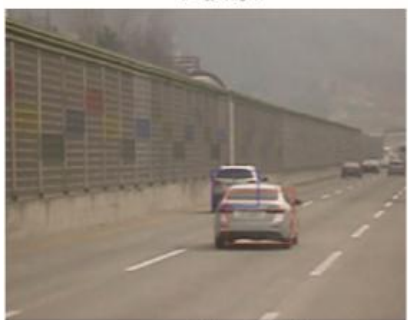

Frame 215

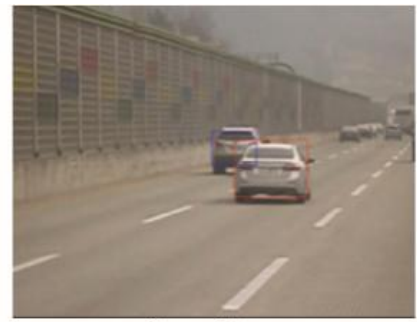

frame 94

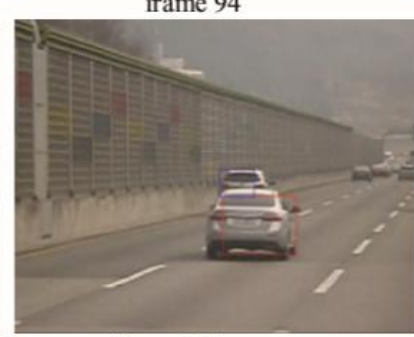

frame 374
Fig.1.Same color vehicle occlusion [39]
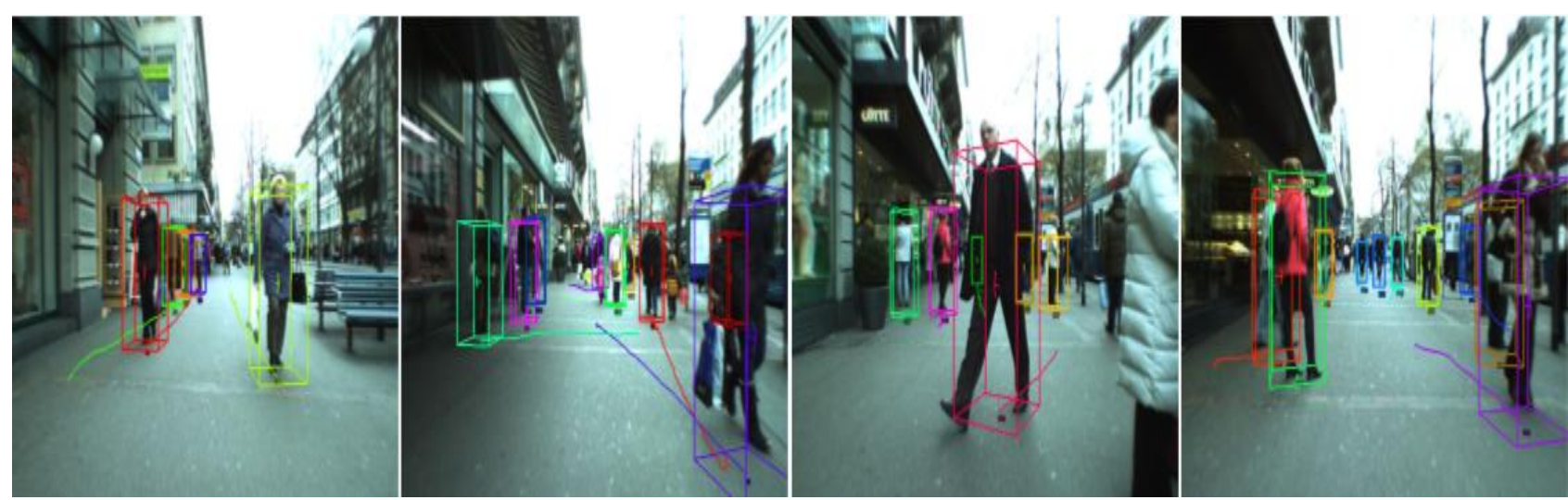

Fig.2.Pedestrian tracking [12] 


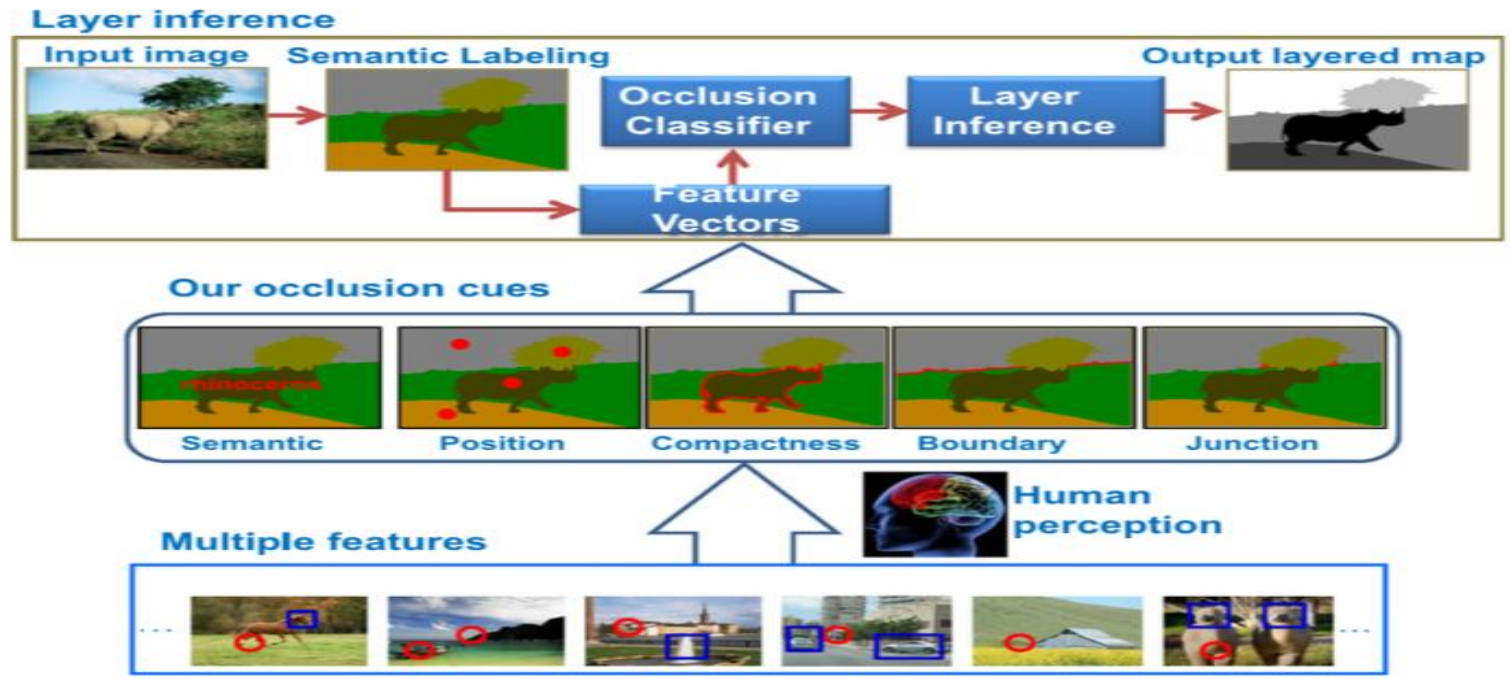

Fig.3.Overview of the method [40]

\section{ACKNOWLEDGMENTS}

Our thanks to the faculty and all the members of lab who have contributed towards development of this survey.

\section{REFERENCES}

[1] Hossein Tehrani Niknejad, Taiki Kawano,Yuki Oishi, Seiichi Mita, "Occlusion Handling using Discriminative Model of Trained Part Templates and Conditional Random Field," 2013 IEEE Intelligent Vehicles Symposium (IV) June 23-26, 2013, Gold Coast, Australia

[2] Andry Maykol Pinto, Paulo G. Costa and A. Paulo Moreira, "An Architecture for Visual Motion Perception of a Surveillance-based Autonomous Robot," 2014IEEEInternational Conference on Autonomous Robot Systems and Competitions(ICARSC) May1415,Espinho,Portugal

[3] Andrej Karpathy Li Fei-Fei, "Andrej Karpathy Li FeiFei," 2014

[4] Whei Zhang,Q.M Jonathan Wu,Xiaokang Yang, "Multilevel framework to detect and handle Occlusion," IEEE Transaction on Intelligent Transport Systems,VOL.9,NO.1,2008

[5] Jiyan Pan,Bo Hu, "Robust Occlusion handling in object tracking," IOP Sciences,Computer Vision and Pattern Recognition,2007

[6] Min Hu, Weiming Hu, Tieniu Tan, "Tracking People through Occlusion," International Conference on Pattern Recognition,2004

[7] M. Zeeshan Zia, Michael Stark and Konrad Schindler, "Are Cars Just 3D Boxes? - Jointly Estimating the 3D Shape of Multiple Objects," IEEE Conference on Computer Vision and Pattern Recognition,2014

[8] Marvin Struwe,Stephan Haslerand Ute Bauer-Wersing, "Using the Analytic Feature Framework for the Detection of Occluded Objects," Artificial Neural Networks and Machine Learning - ICANN 2013
[9] Marvin Struwe1 Stephan Hasler and Ute Bauer-Wersing, "A Two-Stage Classifier Architecture for Detecting Objects under Real-WorldOcclusion Patterns," Artificial Neural Networks and Machine Learning - ICANN 2014

[10] Xiaoyu Wang,Tony X. Han and Shuicheng Yan, "An HOG-LBP Human Detector with Partial Occlusion Handling," IEEE 12th International Conference on Computer Vision (ICCV),2009

[11] M. Zeeshan Zia, Michael Stark and Konrad Schindler, "Explicit Occlusion Modeling for 3D Object Class Representations," IEEE Conference on Computer Vision and Pattern Recognition,2013

[12] A. Ess, K. Schindler, B. Leibe, L. van Gool, "Improved Multi-Person Tracking with Active Occlusion Handling," Proceedings of the IEEE ICRA Workshop on People Detection and Tracking,2009

[13] Jian Sun, Yin Li, Sing Bing Kang, Heung-Yeung Shum, "Symmetric Stereo Matching for Occlusion Handling," Computer Vision and Pattern Recognition, 2005.

[14] Dominik Maximilián Ramík,Christophe Sabourin,Ramon Moreno,Kurosh Madani, "A machine learning based intelligent vision system for autonomous object detection and recognition," Applied Intelligence, Volume 40, Issue 2, pp 358-375, March 2014

[15] Sing Bing Kang, Szeliski R. , Jinxiang Chai, "Handling occlusions in dense multi-view stereo," Computer Vision and Pattern Recognition, 2001

[16] Rashid Mehmood,Rab Nawaz,Naveed Iqbal Rao, "Occlusion Handling in Meanshift Tracking using Adaptive Window Normalized Cross Correlation," 11th International Bhurban Conference on Applied Sciences and Technology (IBCAST), 2014

[17] Yaowen Guan, Xiaoou Chen, Deshun Yang, Yuqian Wu, "Multiperson tracking by detection with local particle filtering and global occlusion handling," IEEE International Conference on Multimedia and Expo (ICME), 2014 
[18] Tao Yang, Stan Z.Li, Quan Pan, Jing Li, "Real-time Multiple Objects Tracking with Occlusion Handling in Dynamic Scenes," IEEE Computer Society Conference on Computer Vision and Pattern Recognition,2005

[19] Dongbo Min and Kwanghoon Sohn, "Cost Aggregation and Occlusion Handling With WLS in Stereo Matching," IEEE Transactions on image processing, VOL. 17, NO. 8,2008

[20] Bing-Fei Wu et al. , "A Relative-DiscriminativeHistogram-of-Oriented-Gradients-Based Particle Filter Approach to Vehicle Occlusion Handling and Tracking," IEEE Transactions on Industrial Electronics, VOL. 61, NO. 8,2014

[21] Markus Enzweiler,Angela Eigenstetter,Bernt Schiele,Dariu M. Gavrila, "Multi-Cue Pedestrian Classification With Partial Occlusion Handling," IEEE Conference on Computer Vision and Pattern Recognition, 2010

[22] Woo-Seok Jang , Yo-Sung Ho, "Efficient depth map generation with occlusion handling for various camera arrays," Signal, Image and Video Processing, Volume 8, Issue 2, pp 287-297,2014

[23] Woo-Seok Jang and Yo-Sung Ho, "Efficient Disparity Map Estimation Using Occlusion Handling for Various 3D Multimedia Applications," IEEE Transactions on Consumer Electronics, 2011

[24] Geoffrey Egnal and Richard P. Wildes, "Detecting Binocular Half-Occlusions:Empirical Comparisons of Five Approaches,'IEEE Transactions on pattern analysis and machine intelligence, VOL. 24, NO. 8, 2002

[25] Christian Wojek, Stefan Walk, Stefan Roth and Bernt Schiele, "Monocular 3D Scene Understanding with Explicit Occlusion Reasoning," IEEE Conference on Computer Vision and Pattern Recognition (CVPR), 2011

[26] Hossein Tehrani Niknejad, Taiki Kawano,Yuki Oishi, Seiichi Mita, "Occlusion Handling using Discriminative Model of Trained Part Templates and Conditional Random Field," IEEE Intelligent Vehicles Symposium ,2013

[27] Brian Fehrman and Jeff McGough,"Handling Occlusion with an Inexpensive Array ofCameras,"IEEE Southwest Symposium on Image Analysis and Interpretation (SSIAI), 2014

[28] P. Wunsch and G. Hirzinger, "Real-Time Visual Tracking of 3-D Objects with Dynamic Handling of
Occlusion," Proceedings of the IEEEInternational Conference on Robotics and Automation,1997

[29] Fabienne Lathuiliere and Jean-Yves Herve, "Visual Tracking of Hand Posture with Occlusion Handling,"15th International Conference on Pattern Recognition, 2000

[30] Ting - Hsun Chang,Shaogang Gong and Eng Jon Ong, "Tracking multiple people under occlusion using multiple cameras," BMVC,2000

[31] Ro'merRosales and StanSclaroff, "Improved Tracking of Multiple Humans with Trajectory Prediction and Occlusion Modeling," IEEE Conf. on Computer Vision and Pattern Recognition, 1998

[32] Andrew Senior et al. , "Appearance models for occlusion handling," Image and Vision Computing ,"VOL.24,2006

[33] Yasuyuki SUGAYA and Yuichi OHTA, "Stereo by the integration of two algorithms with/without occlusion handling,"15th International Conference on Pattern Recognition, 2000

[34] Chenyuan Zhang, Jiu Xu, Axel Beaugendre and Satoshi Goto, "A KLT-Based Approach for Occlusion Handling in Human Tracking,"Picture Coding Symposium,2012

[35] Davi Geiger,Bruce,Ladendorf,Alan Yuille, "Occlusions and Binocular Stereo,'International Journal of Computer Vision, 1995

[36] James J. Little and Walter E. Gillet, "Direct Evidence for Occlusion in Stereo and Motion," Computer Vision - ECCV, 1990

[37] Juyang Weng,Narendra Ahuja,Thomas S. Huang, "Two view matching," IEEE, 1988

[38] Clement Chun Cheong Pang, William Wai Leung Lam, and Nelson Hon Ching Yung,"A Novel Method for Resolving Vehicle Occlusion in a Monocular TrafficImage Sequence,'IEEE Transactions on intelligent transportation systems, VOL. 5, NO. 3,2004

[39] Ming Qing, Van-Dung Hoang and Kang-Hyun Jo Yung,"Localization and Tracking of Same Color Vehicle under Occlusion Proble," Mechatronics-REM, 2012

[40] Xiaowu Chen, Qing li, Dongyue Zhao, Qinping Zhao,"Occlusion cues for image scene layering,"Computer Vision and Image Understanding Volume 117, Issue 1, Pages 42-55, 2013 